\title{
Computability in planar dynamical systems
}

\author{
Daniel Graça \\ DM/FCT, Universidade do Algarve, C. Gambelas, \\ 8005-139 Faro, Portugal \\ \& SQIG /Instituto de Telecomunicações, Lisbon, Portugal \\ email: dgraca@ualg.pt
}

Ning Zhong

DMS, University of Cincinnati,

Cincinnati, OH 45221-0025, U.S.A.

email: ning.zhong@uc.edu

July 24, 2010

\begin{abstract}
In this paper we explore the problem of computing attractors and their respective basins of attraction for continuous-time planar dynamical systems. We consider $C^{1}$ systems and show that stability is in general necessary (but may not be sufficient) to attain computability. In particular, we show that (a) the problem of determining the number of attractors in a given compact set is in general undecidable, even for analytic systems and (b) the attractors are semi-computable for stable systems.

We also show that the basins of attraction are semi-computable if and only if the system is stable.

Keywords: computability, planar dynamical systems, equilibrium points, limit cycles, basins of attraction.
\end{abstract}

\section{Introduction}

Dynamical systems are a powerful tool to model natural phenomena. In essence, a dynamical system is constituted by some phase space, where the dynamics happens, and an evolution law that allows the determination of future states from the present one.

However, inherent to their richness, dynamical systems are mathematically very challenging and not much is known about them, except in some special cases: when the phase state is the plane, when the system is hyperbolic, conservative, etc.

One way to circumvent this problem is to use numerical simulations to get some insight on the dynamical behavior of such systems. However this approach 
usually lacks rigor and therefore formal proofs are still needed. Here we adopt a different perspective: we view computers not only as "approximative tools," but rather as devices which can compute rigourously properties of dynamical systems. Of course, we do not expect that all tasks can be fully automatized. But our aim is to draw the frontier between what is computable and what is not. With this objective in mind we will use the Theory of Computation to establish such results.

In the present paper, we study computability of planar dynamical systems modeled by the differential equation

$$
x^{\prime}=f(x),
$$

where $f: \mathbb{R}^{2} \rightarrow \mathbb{R}^{2}$ is continuously differentiable and $t$ is the independent variable. Our objective is to find out whether or not the attractors and their basins of attraction for planar systems are computable. A basic tool for understanding planar dynamical systems is the Poincaré-Bendixson theorem, which gives a criterion for the detection of limit cycles in the plane. This theorem also plays an important role in the computation of limit cycles in structurally stable planar system.

A point that we would like to emphasize throughout this paper is our belief that some sort of stability is needed (but not sufficient) in a dynamical system to guarantee the computability of its main dynamical properties/structures. The intuition behind this claim it that if some robustness for perturbations is not accounted for, it is impossible to simulate a continuous system with digital computation, where approximation errors are always present. Probably the strongest forms of stability are not required in general, e.g. structural stability. For instance Lorenz attractor [Lor63] is not structurally stable, but it is stable to small perturbations in the parameters defining it [Via00].

However, for planar systems, the meaningful notion of stability is structural stability as shown by Peixoto's theorem and other results, and will be the notion used here. We will establish results about important structures (equilibrium points, periodic orbits, basins of attraction) for both the general case of (1), and the restricted case of structurally stable system.

This paper is an improved journal version, with new results, of the conference paper [GZ09].

A number of papers have already been written about computability over dynamical systems. For instance, the papers [GZB09], [CG09] show that solutions (trajectories) of (1) must be computable over their lifespan (the maximal interval on which the solution exists), under the classical conditions ensuring existence of a solution to (1) for a given initial point. In [GZB09] one can also find results about the non-computability of lifespan of trajectories for analytic functions $f$, with further generalizations presented in [GCB09], [RWZ09].

In [Zho09], it is shown that the domain of attraction of a computable and asymptotically stable hyperbolic equilibrium point of the nonlinear system (1) is in general not computable, though it is semi-computable. This tells us that the domain of attraction can be approximated from the inside on the one hand, 
but on the other hand there is no algorithm determining how far such an approximation is from filling up this domain.

Other related results can be found in, e.g. [Moo90], [BBKT01], [Col05], [BY06], [Hoy07]. See [BGZed] for a more detailed review of the literature.

\section{Differential equations}

This section provides a summary of results concerning the ODE (1) with a focus on the planar case. We assume that $f$ is continuously differentiable on $\mathbb{R}^{2}$, i.e., $f \in C^{1}\left(\mathbb{R}^{2}\right)$. For more details the reader is referred to [HS74], [GH83], and [Per01].

Definition 1 Let $\phi\left(t, x_{0}\right)$ denote the solution of (1) corresponding to the initial condition $x(0)=x_{0}$. The function $\phi\left(\cdot, x_{0}\right): \mathbb{R} \rightarrow \mathbb{R}^{2}$ is called a solution curve, trajectory, or orbit of (1) through the point $x_{0}$.

1. A point $x$ is called an equilibrium point of (1) if $f(x)=0$. An equilibrium point $x_{0}$ is called hyperbolic if none of the eigenvalues of the gradient matrix $D f\left(x_{0}\right)$ of $f$ at $x_{0}$ has zero real part.

2. An equilibrium point $x_{0}$ of (1) is called stable if for any $\varepsilon>0$, there exists $a \delta>0$ such that $\left|\phi(t, \tilde{x})-x_{0}\right|<\varepsilon$ for all $t \geq 0$, provided $\left|\tilde{x}-x_{0}\right|<\delta$. Furthermore, $x_{0}$ is called asymptotically stable if it is stable and there exists a (fixed) $\delta_{0}>0$ such that $\lim _{t \rightarrow \infty} \phi(t, \tilde{x})=x_{0}$ for all $\tilde{x}$ satisfying $\left|\tilde{x}-x_{0}\right|<\delta_{0}$.

Given a trajectory $\Gamma_{x_{0}}=\phi\left(\cdot, x_{0}\right)$, we define the positive half-trajectory as $\Gamma_{x_{0}}^{+}=\left\{\phi\left(t, x_{0}\right) \mid t \geq 0\right\}$. When the context is clear, we often drop the subscript $x_{0}$ and write simply $\Gamma$ and $\Gamma^{+}$.

It is not difficult to see that if $x_{0}$ is an equilibrium point of $(1)$, then $\phi\left(t, x_{0}\right)=$ $x_{0}$ for all $t \geq 0$. It is also known that if the real parts of all eigenvalues of $D f\left(x_{0}\right)$ of a hyperbolic equilibrium point $x_{0}$ are negative, then $x_{0}$ is asymptotically stable; in this case $x_{0}$ is called a sink. Other types of hyperbolic equilibria are sources (the real parts of all eigenvalues of $D f\left(x_{0}\right)$ are positive) and saddles (the real parts of the eigenvalues of $D f\left(x_{0}\right)$ have mixed signs). A source becomes a sink under the time variable change $\bar{t}=-t$, i.e. sources are asymptotically stable when $t \rightarrow-\infty$.

Next we define limit sets and limit cycles.

Definition 2 1. A point $p \in \mathbb{R}^{2}$ is an $\omega$-limit ( $\alpha$-limit) point of the trajectory $\phi(\cdot, x)$ of the system (1) if there is a sequence $t_{n} \rightarrow \infty\left(t_{n} \rightarrow-\infty\right)$ such that $\lim _{n \rightarrow \infty} \phi\left(t_{n}, x\right)=p$.

Definition 3 The set of all $\omega$-limit ( $\alpha$-limit) points of the trajectory $\Gamma$ is called the $\omega$-limit ( $\alpha$-limit) set of $\Gamma$; written as $\omega(\Gamma)$ or $\omega(x)(\alpha(\Gamma)$ or $\alpha(x))$ if $\Gamma=$ $\phi(\cdot, x)$. 
Definition $4 A$ cycle or periodic orbit of (1) is any closed solution curve of (1) which is not an equilibrium point. A cycle $\Upsilon$ is stable if for each $\varepsilon>0$ there is a neighborhood $U$ of $\Upsilon$ such that for all $x \in U, d\left(\Gamma_{x}^{+}, \Upsilon\right)<\varepsilon$. A cycle $\Upsilon$ is asymptotically stable if for all points $x_{0}$ in some neighborhood $U$ of $\Upsilon$ one has $\lim _{t \rightarrow \infty} d\left(\phi\left(t, x_{0}\right), \Upsilon\right)=0$.

Definition 5 A limit cycle is a periodic orbit $\Gamma$ which is the $\alpha$ or $\omega$-limit set of some trajectory of (1) other than $\Gamma$.

We can define hyperbolic periodic orbits in terms of characteristics exponents, by means of the Stable Manifold Theorem for periodic orbits (see e.g. [Per01, Section 3.5]), in a way similar to Definition 1. However, to avoid technical issues not relevant to our discussion, we simply state their properties interest to our case (see [Per01, Section 3.5]): in a sufficiently small neighborhood, the convergence rate to any hyperbolic limit cycle is exponential $\left(\beta e^{-\lambda t}\right.$, with $\beta>0$ and $\lambda \neq 0$ ). Hyperbolic periodic orbits can be attracting (when $\lambda>0$ ) or repelling (when $\lambda<0$ ).

Definition $6 A$ set $A \subseteq \mathbb{R}^{2}$ is invariant if $\phi(t, x) \in A$ for all $x \in A$ and $t \in[0,+\infty)$. If $A$ is a closed invariant set, its domain of attraction (or basin of attraction, or simply basin of $A$ ) is the set

$$
\left\{x \in \mathbb{R}^{2}: \omega(x) \subseteq A\right\} .
$$

Domains of attraction are separated by curves in $\mathbb{R}^{2}$ (stable and unstable manifolds - see [GH83, p. 34]) and therefore are open sets.

The following result can be found in e.g. [Per01].

Proposition 7 (The Poincaré-Bendixson Theorem) Suppose that $f \in C^{1}(E)$ where $E \subseteq \mathbb{R}^{2}$ is open and that (1) has a trajectory $\Gamma$ such that $\Gamma^{+}$is contained in a compact subset $M \subseteq E$. Then if $\omega(\Gamma)$ contains no singularity of $(1), \omega(\Gamma)$ is a periodic orbit of (1).

For this paper it will be of special relevance to consider structurally stable dynamical systems. A dynamical system defined by (1) is structurally stable if for any vector field $g$ close to $f$, the vector fields $f$ and $g$ are topologically equivalent (see [HS74], [Per01] for further details). In practice, this means that if we perturb the system (1) by a small amount, the resulting dynamical system will stay close to the one defined by (1). The following results are classical and can be found e.g. in [Per01].

Proposition 8 (Peixoto's theorem) If $M \subseteq \mathbb{R}^{2}$ is compact, then the set of structurally stable vector fields in $C^{1}(M)$ is an open, dense subset of $C^{1}(M)$.

Proposition 9 Given a structurally stable dynamical system defined on a compact subset $M \subseteq \mathbb{R}^{2}$, its only limit sets are hyperbolic equilibrium points and hyperbolic periodic orbits, and these appear in a finite number. 
Finally we present some estimates on the error committed in the computation of a trajectory when the system is perturbed. Let $x$ be a solution of the equation (1). Let $y$ be a solution of the ODE

$$
y^{\prime}=g(y) .
$$

The following result is classical and can be found in e.g. [HS74]

Lemma 10 In the above conditions, and supposing that $x$ and $y$ are defined on a region $\mathcal{D}$ where $f$ and $g$ satisfy a Lipschitz condition, with Lipschitz constant $L$, then on $\mathcal{D}$

$$
\|x(t)-y(t)\| \leq\|x(0)-y(0)\| e^{L t}+\frac{\varepsilon}{L}\left(e^{L t}-1\right),
$$

provided $\|f-g\| \leq \varepsilon$.

We also set up the following notation. A trajectory $\phi: \mathbb{R}_{0}^{+} \rightarrow \mathbb{R}^{2}$ is a solution of the differential inclusion

$$
x^{\prime} \in f_{\varepsilon}(x),
$$

where $f_{\varepsilon}$ is a set-valued function defined by $f_{\varepsilon}(x)=B(f(x), \varepsilon)$ (the ball of center $f(x)$ and radius $\varepsilon)$ if $\phi^{\prime}(t) \in f_{\varepsilon}(\phi(t))$ almost everywhere. See [Dei84], [AC84] for further details on differential inclusions.

\section{Computable analysis}

This section introduces necessary concepts and results from computable analysis which are needed for this paper. For more details the reader is referred to [PER89], [Ko91], [Wei00]. The idea underlying computable analysis to compute over a set $A$ is to encode each element $a$ of $A$ by a countable sequence of symbols from a finite alphabet (called a $\rho$-name of $a$ ). Each sequence can encode at most one element of $A$. The more elements we have from a sequence encoding $a$, the more precisely we can pinpoint $a$. From this point of view, it suffices to work only with $\rho$-names when performing computation over $A$. To compute with $\rho$ names, we use Type-2 machines, which are similar to Turing machines, but (i) have a read-only tape, where the input (i.e. the sequence encoding it) is written; (ii) have a write-only output tape, where the head cannot move back and the sequence encoding the output is written.

At any finite amount of time we can halt the computation, and we will have a partial result on the output tape. The more time we wait, the more accurate this result will be. We now introduce notions of computability over $\mathbb{R}^{l}$.

Definition 11 1. A sequence $\left\{r_{n}\right\}$ of rational numbers is called a $\rho$-name of a real number $x$ if there are three functions $a, b$ and $c$ from $\mathbb{N}$ to $\mathbb{N}$ such that for all $n \in \mathbb{N}, r_{n}=(-1)^{a(n)} \frac{b(n)}{c(n)+1}$ and

$$
\left|r_{n}-x\right| \leq \frac{1}{2^{n}}
$$


2. A double sequence $\left\{r_{n, k}\right\}_{n, k \in \mathbb{N}}$ of rational numbers is called a $\rho$-name for a sequence $\left\{x_{n}\right\}_{n \in \mathbb{N}}$ of real numbers if there are three functions a, $b, c$ from $\mathbb{N}^{2}$ to $\mathbb{N}$ such that, for all $k, n \in \mathbb{N}, r_{n, k}=(-1)^{a(k, n)} \frac{b(k, n)}{c(k, n)+1}$ and

$$
\left|r_{n, k}-x_{n}\right| \leq \frac{1}{2^{k}}
$$

3. A real number $x$ (a sequence $\left\{x_{n}\right\}_{n \in \mathbb{N}}$ of real numbers) is called computable if it has a computable $\rho$-name, i.e. the functions $a, b$, and $c$ are computable or, equivalently, there is a Type-2 machine that computes a $\rho$-name of $x$ without any input.

The notion of the $\rho$-name can be extended to points in $\mathbb{R}^{l}$ as follows: a sequence $\left\{\left(r_{1 n}, r_{2 n}, \ldots, r_{l n}\right)\right\}_{n \in \mathbb{N}}$ of rational vectors is called a $\rho$-name of $x=$ $\left(x_{1}, x_{2}, \ldots, x_{l}\right) \in \mathbb{R}^{l}$ if $\left\{r_{j n}\right\}_{n \in \mathbb{N}}$ is a $\rho$-name of $x_{j}, 1 \leq j \leq l$. Having $\rho$-names, we can define computable functions.

Definition 12 Let $A, B$ be sets, where $\rho$-names can be defined for elements of $A$ and $B$. A function $f: A \rightarrow B$ is computable if there is a Type-2 machine such that on any $\rho$-name of $x \in A$, the machine computes as output a $\rho$-name of $f(x) \in B$.

Next we present a notion of computability for open and closed subsets of $\mathbb{R}^{l}$ (cf. [Wei00], Definition 5.1.15). We implicitly use $\rho$-names - sequences of symbols (or rationals in this case, which can easily be encoded as finite strings) which encode a given object - see [Wei00] for further details. For instance, to obtain names of open subsets of $\mathbb{R}^{l}$, we note that the set of rational balls $B(a, r)=\left\{x \in \mathbb{R}^{l}:|x-a|<r\right\}$, where $a \in \mathbb{Q}^{l}$ and $r \in \mathbb{Q}$, is a basis for the standard topology over $\mathbb{R}^{l}$. Thus a sequence $\left\{\left(a_{n}, r_{n}\right)\right\}_{n \in \mathbb{N}}$ such that $E=\cup_{n=0}^{\infty} B\left(a_{n}, r_{n}\right)$ gives a $\rho$-name for $E$. Depending on the $\rho$-names used, we obtain different notions of computability. We omit further details for reasons of space.

Definition 13 1. An open set $E \subseteq \mathbb{R}^{l}$ is called recursively enumerable (r.e. for short) open if there are computable sequences $\left\{a_{n}\right\}$ and $\left\{r_{n}\right\}, a_{n} \in \mathbb{Q}^{l}$ and $r_{n} \in \mathbb{Q}$, such that

$$
E=\cup_{n=0}^{\infty} B\left(a_{n}, r_{n}\right) .
$$

Without loss of generality one can also assume that for any $n \in \mathbb{N}$, the closure of $B\left(a_{n}, r_{n}\right)$, denoted as $\overline{B\left(a_{n}, r_{n}\right)}$, is contained in $E$.

2. A closed subset $K \subseteq \mathbb{R}^{l}$ is called r.e. closed if there exist computable sequences $\left\{b_{n}\right\}$ and $\left\{s_{n}\right\}, b_{n} \in \mathbb{Q}^{l}$ and $s_{n} \in \mathbb{Q}$, such that $\left\{B\left(b_{n}, s_{n}\right)\right\}_{n \in \mathbb{N}}$ lists all rational open balls intersecting $K$.

3. An open set $E \subseteq \mathbb{R}^{l}$ is called computable (or recursive) if $E$ is r.e. open and its complement $E^{c}$ is r.e. closed. Similarly, a closed set $K \subseteq \mathbb{R}^{l}$ is called computable (or recursive) if $K$ is r.e. closed and its complement $K^{c}$ is r.e. open. 
4. A compact set $M \subseteq \mathbb{R}^{l}$ is called computable if it is computable as a closed set and, in addition, there is a rational number $b$ such that $\|x\| \leq b$ for all $x \in M$.

When dealing with open sets in $\mathbb{R}^{l}$, we identify a special type of computability, that we call semi-computability. Let $\mathcal{O}\left(\mathbb{R}^{l}\right)=\left\{O \mid O \subseteq \mathbb{R}^{l}\right.$ is open in the standard topology\}.

Definition 14 function $f: A \rightarrow \mathcal{O}\left(\mathbb{R}^{l}\right)$ is called semi-computable if there is a Type-2 machine such that on any $\rho$-name of $x \in A$, the machine computes as output two sequences $\left\{a_{n}\right\}$ and $\left\{r_{n}\right\}, a_{n} \in \mathbb{Q}^{l}$ and $r_{n} \in \mathbb{Q}$, such that

$$
f(x)=\cup_{n=0}^{\infty} B\left(a_{n}, r_{n}\right) .
$$

Without loss of generality one can also assume that for any $n \in \mathbb{N}$, the closure of $B\left(a_{n}, r_{n}\right)$ is contained in $f(x)$.

We call this function semi-computable because we can tell in a finite amount of time if a point belongs to $f(x)$, but we have to wait an infinite amount of time to know that it does not belong to $f(x)$.

In the rest of the paper, we will work exclusively with $C^{1}$ functions $f: E \rightarrow$ $\mathbb{R}^{2}$, where $E$ is an open subset of $\mathbb{R}^{2}$. Thus it is desirable to present an explicit $\rho$-name for such functions.

Definition 15 Let $E=\cup_{n=0}^{\infty} B\left(a_{n}, r_{n}\right), a_{n} \in \mathbb{Q}^{2}$ and $r_{n} \in \mathbb{Q}$, be an open subset of $\mathbb{R}^{2}$ (assuming that the closure of $B\left(a_{n}, r_{n}\right)$ is contained in $E$ ) and let $f: E \rightarrow \mathbb{R}^{2}$ be a continuously differentiable function. Then a $\left(C^{1}\right) \rho$-name of $f$ is a sequence $\left\{P_{k}\right\}$ of polynomials $\left(P_{k}: \mathbb{R}^{2} \rightarrow \mathbb{R}^{2}\right)$ with rational coefficients such that

$$
d_{C^{1}(E)}\left(f, P_{k}\right) \leq 2^{-k} \quad \text { for all } k \in \mathbb{N}
$$

where

$$
d_{C^{1}(E)}\left(f, P_{k}\right)=\sum_{n=0}^{\infty} 2^{-n}\left(\frac{\left\|f-P_{k}\right\|_{n}}{1+\left\|f-P_{k}\right\|_{n}}+\frac{\left\|D f-D P_{k}\right\|_{n}}{1+\left\|D f-D P_{k}\right\|_{n}}\right)
$$

and

$$
\|g\|_{n}=\max _{x \in \overline{B\left(a_{n}, r_{n}\right)}}|g(x)| .
$$

We observe that this $\rho$-name of $f$ contains information on both $f$ and $D f$ in the sense that $\left(P_{1}, P_{2}, \ldots\right)$ is a $\rho$-name of $f$ while $\left(D P_{1}, D P_{2}, \ldots\right)$ is a $\rho$-name of $D f$. See [ZW03] for further details.

Throughout the rest of this paper, unless otherwise mentioned, we will assume that, in (1), $f$ is continuously differentiable on an open subset of $\mathbb{R}^{2}$ and we will use the above $\rho$-name for $f$.

Before closing this section, we present some useful results from [GZB09]. Recall that a function $f: E \rightarrow \mathbb{R}^{m}, E \subseteq \mathbb{R}^{l}$, is said to be locally Lipschitz on $E$ if it satisfies a Lipschitz condition on every compact set $V \subset E$. The following definition gives a computable analysis analog of this condition (which is an extension of the homonymous definition presented in [GZB09]). 
Definition 16 Let $E=\cup_{n=0}^{\infty} B\left(a_{n}, r_{n}\right) \subseteq \mathbb{R}^{l}$, where $\overline{B\left(a_{n}, r_{n}\right)} \subseteq E$, be an open set. A function $f: E \rightarrow \mathbb{R}^{m}$ is called effectively locally Lipschitz on $E$, if from $\rho$-names of $f$ and $E$ one can compute a sequence $\left\{K_{n}\right\}$ of positive integers such that

$$
|f(x)-f(y)| \leq K_{n}|x-y| \text { whenever } x, y \in \overline{B\left(a_{n}, r_{n}\right)} .
$$

Theorem 17 Assume that $f: E \rightarrow \mathbb{R}^{m}$ is a $C^{1}$ function. Then $f$ is effectively locally Lipschitz.

Proof. The proof is essentially the proof of [GZB09, Theorem 2.7].

\section{Main algorithm}

This section presents the foundations of the construction which will be used throughout the remaining of this paper. The objective of this construction is to discretize the dynamics of (1). Access to a $\left(C^{1}\right) \rho$-name of $f$ is sufficient for the construction. We begin by dividing the state space into a finite number of pieces, which corresponds to the vertices of a directed graph. One is able to go from one vertex $A$ to another vertex $B$, if the flow starting on $A$ will reach vertex $B$. This allows one to analyze the system in finite time. However, in order to be able to compute the edges of the graph, some positive error in the right-hand-side of (1) has to be introduced though this error may be as small as desired.

This procedure is an adaptation (we restrict ourselves to differential equations and not inclusions) and extension (this construction works for unbounded domains) of a technique introduced in [PBV95]. This latter technique has two limitations: (i) it only works on compact spaces; (ii) the flow must be pointing inwards at the boundary of this compact set (i.e. the flow cannot leave the compact if the algorithm is to provide an accurate computation. This condition is not explicitly stated on the paper but it is implicitly used). Our idea of overcoming these difficulties can be, roughly speaking, summarized as follows:

1. Repeatedly execute a subroutine starting from $n=1$, and increment $n$ at each repetition.

2. Each time the subroutine is executed, pick an $n \times n$-square centered at the origin (more precisely a $2 n \times 2 n$-square, but for simplicity we omit linear factors), compute some values (Lipschitz constant) and apply the algorithm from [PBV95] using the following parameters: (i) each finite piece of the square has diameter $\leq \frac{1}{n}$; (ii) the positive error in the computation of the right-hand side of (1) is bounded by $\frac{1}{n}$.

3. Each time the flow leaves the $n \times n$-square, mark the respective pre-image pieces with a special symbol $\mathbf{p}$, meaning that the flow could not be fully computed from these pieces. 


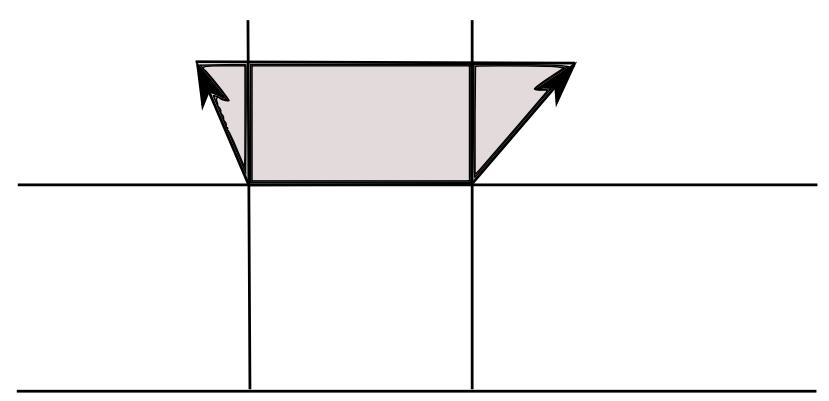

Figure 1: Flow from a $\frac{1}{n} \times \frac{1}{n}$-square. In the figure it is only represented the flow for the upper face of the square (for other faces the flow would have the same direction, but would leave from that face. See Fig. 4.)

We will see that this procedure is sufficient to compute dynamical properties for structurally stable systems. On the other hand, we will show that no algorithm exists for computing those properties over structurally unstable systems.

We now describe the algorithm in more details. For some value $n$ fixed in the first step, define $S_{n}=(-n, n)^{2}$ as the square mentioned in step 2. Let $L_{n} \in \mathbb{N}$ be a rational Lipschitz constant valid over $S_{n}$, which can be computed from $n$ by Theorem 17 . Now divide $S_{n}$ into small pieces - squares of size $\frac{1}{2 n L_{n}} \times \frac{1}{2 n L_{n}}$, which we call $\frac{1}{n} \times \frac{1}{n}$-squares.

Let $s$ be one of these squares. Since the closure of $s, c l(s)$, is compact, one can compute in finite time (see e.g. [Wei00]) a rational polytope $P_{s, n}$ (polytope of directions) such that $f(s) \subseteq P_{s, n}$ (it is an over-approximation of $f(s)$ ) and $\operatorname{dist}\left(f(s), P_{s, n}\right)<\frac{1}{n}$, where $\operatorname{dist}\left(f(s), P_{s, n}\right)$ is the Hausdorff distance between $f(s)$ and $P_{s, n}$.

Now we define a function $\Theta$ over the set of all $\frac{1}{n} \times \frac{1}{n}$ squares $s$ as $\Theta(s)=\bigcup A_{s}$, where $A_{s}$ is the collection of all $\frac{1}{n} \times \frac{1}{n}$ squares which are adjacent to one of the faces of $s$ (not including $s$ ) and intersect with the following rational polytope

$$
R_{s, n}=\left\{\alpha+t P_{s, n} \mid t \in \mathbb{R}_{0}^{+}, \alpha \text { is a face of } s\right\} .
$$

(cf. Fig. 1). Since $P_{s, n}$ is rational, as well as the endpoints of each face $\alpha$ of $s$, $\Theta(s)$ can be computed in finite time (there are only a finite number of adjacent squares to $s$ )

There is a special case to be dealt with: $\alpha$ is part of the boundary of $S_{n}$ (i.e. $s$ is not completely surrounded by $\frac{1}{n} \times \frac{1}{n}$ squares). In this case we check if the set (3) leaves the face $\alpha$ and goes (partially or not) to the set $\overline{S_{n}}$, the complement of $S_{n}$. If this is the case, proceed as before, i.e. continue the "trajectory" remaining inside $S_{n}$ but don't add $\frac{1}{n} \times \frac{1}{n}$ squares outside of $S_{n}$ to $\Theta(s)$, marking $s$ with some symbol, say $\$$. This indicates that the "trajectory" is incomplete from this point, since it leaves $S_{n}$. 
Given a $\frac{1}{n} \times \frac{1}{n}$ square $s \subseteq S_{n}$, its $n$-polygonal trajectory is $\operatorname{Traj}_{n}(s)=$ $\cup_{i=0}^{\infty} \Theta^{i}(s)$ which can be computed in finite time by the following sketch of algorithm:

$$
\begin{aligned}
& R_{0}=s \\
& R_{i+1}=R_{i} \cup \Theta\left(R_{i}\right)
\end{aligned}
$$

The halting condition of the algorithm is $R_{i+1}=R_{i}$, meaning that the polygonal trajectory cannot go further inside $S_{n}$. The computation will halt in a finite number of steps, since $R_{i} \subseteq R_{i+1}$ and the number of $\frac{1}{n} \times \frac{1}{n}$ squares in $S_{n}$ is finite. In other words, $\operatorname{Traj}_{n}(s)=R_{j}$ for some $j \in \mathbb{N}$. In any step, if $\Theta\left(R_{i}\right)$ is marked with $\mathbf{p}$, then also mark $s$ with $\boldsymbol{\beta}$ to indicate that $\operatorname{Traj}_{n}(s)$ is incomplete (it goes out of the square $S_{n}$ ). The following lemma follows from the described construction.

Lemma 18 Let $s$ be a $\frac{1}{n} \times \frac{1}{n}$ square as defined above. If $\phi(t, s) \in S_{n}$ for all $t \in[0, T)$, where $0 \leq T \leq+\infty$, then

1. $\{\phi(t, s) \mid t \in[0, T)\} \subseteq \operatorname{Traj}_{n}(s)$, and

2. for each $\varepsilon>0$ there exists $n_{0} \in \mathbb{N}$ such that for every $n \geq n_{0}, \operatorname{Traj}_{n}(s) \subseteq$ $\left\{\phi(t, s) \mid \phi^{\prime}(t) \in f_{\varepsilon}(\phi(t))\right\}$.

Proof. For claim 1 let $x \in s$. By definition of $\operatorname{Traj}_{n}(s)$, it is clear that as long as $\phi(t, x) \in s$ one must have $\phi(t, x) \in \operatorname{Traj}_{n}(s)$. Now suppose that at some time $t_{\alpha}, \phi(t, x)$ leaves $s$ to enter a square $s^{\prime}$. Since $f\left(\phi\left(t_{\alpha}, x\right)\right) \in f(s) \subseteq P_{s, n}$, it is obvious that $s^{\prime} \in \Theta(s)$. Therefore, as long as the trajectory remains inside $s^{\prime}$, one has $\phi(t, x) \in \operatorname{Traj}_{n}(s)$. Proceeding recursively, we conclude claim 1 .

For claim 2, consider a $\frac{1}{n} \times \frac{1}{n}$ square $s$. Since $s$ is a square of size $\frac{1}{2 L_{n} n} \times \frac{1}{2 L_{n} n}$, two points $x, y \in s$ are at most within distance $\frac{\sqrt{2}}{2 L_{n} n} \leq \frac{1}{L_{n} n}$. Since $L_{n}$ is a Lipschitz constant, $|f(x)-f(y)| \leq \frac{1}{n}$. This implies that for any $x \in s$, if one picks an $n_{0}$ such that $\frac{2}{n_{0}} \leq \varepsilon$, then

$$
f(s) \subseteq P_{s, n_{0}} \subseteq f_{\varepsilon}(x)
$$

Thus, while on square $s$, claim 2 is true. If $\operatorname{Traj}_{n_{0}}(s)$ leaves $s$ to enter a square $s^{\prime}$, it follows from (4) that the trajectory cone $\left\{\phi(t, s) \mid \phi^{\prime}(t) \in f_{\varepsilon}(\phi(t))\right\}$ also enters $s^{\prime}$. Let $z$ be a point belonging to this trajectory cone. But then (4) will also hold in $s^{\prime}$ and thus claim 2 is also true while on square $s^{\prime}$. Again, by recursion, we conclude claim 2 .

Some simple facts can be obtained from this construction:

Lemma 19 Assume conditions as above:

1. Let $\gamma \subseteq S_{n}$ be a limit cycle. If $s$ intersects $\gamma$ and $\gamma \nsubseteq s$ then there is some $i>0$ such that $s \subseteq \Theta\left(R_{i}-s\right)$.

2. If $\operatorname{Traj}_{n}(s)$ is not marked with $\boldsymbol{\beta}$, then: 
(a) If $\operatorname{Traj}_{n}(s)$ doesn't include any square containing a sink $p$, then $s$ is not contained in the basin of attraction of $p$.

(b) If $\operatorname{Traj}_{n}(s)$ doesn't include any square intersecting a limit cycle $\gamma$, then $s$ is not contained in the basin of attraction of $\gamma$.

(c) If $\operatorname{Traj}_{n}(s)$ includes exactly one square containing a sink $p$ and does not include any limit cycle, then $s$ is in the basin of attraction of $p$.

(d) If $\operatorname{Traj}_{n}(s)$ doesn't include any square containing an equilibrium point, and includes squares that intersect only one limit cycle $\gamma$, then $s$ is in the basin of attraction of $\gamma$.

3. If $E \subseteq S_{n}$ is a union of $\frac{1}{n} \times \frac{1}{n}$ squares such that $\operatorname{Traj}_{n}(E)$ is not marked with $\$$ and $\operatorname{Traj}_{n}(E)=E$, then $E$ is invariant set.

Proof. For claim 1, note that the flow will leave $s$, but it will reenter $s$ again. For claims 2-(a) \& 2-(b), it suffices to notice that (i) $\operatorname{Traj}_{n}(s)$ is an overapproximation of the real flow starting on $s$; (ii) the flow doesn't go to infinity (this is the case because $\operatorname{Traj}_{n}(s)$ is not marked with $\mathbf{p}$ ), and thus must converge to some equilibria and/or limit cycles (notice that the flow originating on $s$ may converge to multiple equilibria/limit cycles).

From the preceding observations, it is clear that if $\operatorname{Traj}_{n}(s)$ converges to only one equilibrium point/limit cycle and does not leave $S_{n}$, then the same must happen to the flow originating on $s$, thus ensuring that $s$ belongs to the basin of attraction of the equilibrium point/limit cycle. This proves claims 2-(c) $\& 2-(\mathrm{d})$.

For claim 3, if $\operatorname{Traj}_{n}(E)=E$, then by Lemma 18 it follows that $\{\phi(t, E) \mid t \in$ $[0,+\infty)\} \subseteq E$ and, consequently, $E$ is an invariant set.

\section{Computing attractors}

By Proposition 9, in structurally stable planar systems, equilibria can only be of two types: hyperbolic fixed points and hyperbolic periodic orbits, and these appear in a finite number on a compact set.

\subsection{Computing fixed points}

Fixed points are zeros of (1) and it is a classical result that in general the zeros cannot be computed from $f$-see e.g. [Wei00]. Therefore it is natural that one cannot compute the number of equilibrium points in (1). That's exactly what the next theorem proves.

Theorem 20 Given as input a $\rho$-name of an analytic function $f$, the problem of deciding the number of equilibrium points in (1) is undecidable, even on compact sets. 
Proof. Let $T M_{1}, \cdots, T M_{n}, \ldots$ be an enumeration of all Turing machines, and consider the computable function $g: \mathbb{N}^{2} \rightarrow \mathbb{N}$ defined by

$$
g(k, i)= \begin{cases}0 & \text { if } T M_{k} \text { stops in } \leq i \text { steps with input } k \\ 1 & \text { otherwise. }\end{cases}
$$

Then $\sum_{i=1}^{\infty} \frac{g(k, i)}{2^{i}}$ is a computable number with the property that $\sum_{i=1}^{\infty} \frac{g(k, i)}{2^{i}}<1$ if $T M_{k}$ halts on input $k, \sum_{i=1}^{\infty} \frac{g(k, i)}{2^{i}}=1$ otherwise. Define $f: \mathbb{R}^{2} \rightarrow \mathbb{R}^{2}$ in (1) such that both components of $f$ are the computable function

$$
h_{k}(x, y)=\left(x-\sum_{i=1}^{\infty} \frac{g(k, i)}{2^{i}}\right)(x-1)+y^{2}
$$

We observe that $f$ is an analytic function. It is easy to see that the system (1) admits two distinct equilibria $\left(\sum_{i=1}^{\infty} \frac{g(k, i)}{2^{i}}, 0\right)$ and $(1,0)$ if $T M_{k}$ halts on input $k$, and only one equilibrium $(1,0)$ if $T M_{k}$ does not halt on input $k$. Therefore, if we could decide the number of equilibria in (1), then we would be able to decide the problem "does $T M_{k}$ halts on input $k$ ?" which is well-known to be undecidable [Odi89, Th. II.2.3].

However, one can semi-compute the equilibrium points of (1).

Theorem 21 Let $K \subseteq \mathbb{R}^{2}$ be a computable compact set and let $K_{\text {eq }}$ be the set of equilibrium points of (1) in $K$, where $f \in C^{1}(E)$ on some open subset $E \subseteq \mathbb{R}^{2}$ containing $K$. Then, given as input a $\rho$-name of $f$, one can compute a sequence of closed sets $\left\{K_{\text {eq }}^{n}\right\}_{n \in \mathbb{N}}$ with the following properties:

1. $K_{e q}^{n} \subseteq K$ for every $n \in \mathbb{N}$.

2. $K_{e q}^{n+1} \subseteq K_{e q}^{n}$ for every $n \in \mathbb{N}$.

3. $\lim _{n \rightarrow \infty} K_{e q}^{n}=K_{e q}$.

Proof. Using the construction of Section 4 , we can compute a rational polytope $P_{s, n}$ such that $f(s) \subseteq P_{s, n}$ and $\operatorname{dist}\left(f(s), P_{s, n}\right)<\frac{1}{n}$, where $\operatorname{dist}\left(f(s), P_{s, n}\right)$ is the Hausdorff distance between $f(s)$ and $P_{s, n}$. Note that if $f$ has a zero in any $\frac{1}{n_{0}} \times \frac{1}{n_{0}}$ square $s$, then the origin 0 of $\mathbb{R}^{2}$ is contained in $P_{s, n_{0}}$. (Note that we use 0 to denote both the number 0 and the origin of $\mathbb{R}^{2}$.) On the other hand, if $s$ does not contain any zero of $f$, then $\min _{x \in s}\|f(x)\|=\varepsilon>0$ and subsequently, for all $n>\frac{1}{\varepsilon}, P_{s, n_{0}}$ (or more precisely, $P_{s^{\prime}, n}$ for all the squares $s^{\prime}$ covering $s$ for $n \geq n_{0}$ ) does not contain 0 . Since it can be decided whether or not $P_{s, n}$ contains 0 , the following algorithm provides the succession $K_{e q}^{n}$ with the desired properties: For each $n \in \mathbb{N}$ do:

1 Let $n_{0}=n$ (see below why this is necessary). If $K \nsubseteq S_{n_{0}}$, then pick $K_{e q}^{n}=K$; else:

1.1 Start with $K_{e q}^{n}=\varnothing$. For each $\frac{1}{n_{0}} \times \frac{1}{n_{0}}$ square $s$, compute $P_{s, n_{0}}$. If $P_{s, n_{0}}$ contains 0 , then $K_{e q}^{n}=K_{e q}^{n} \cup s$. 


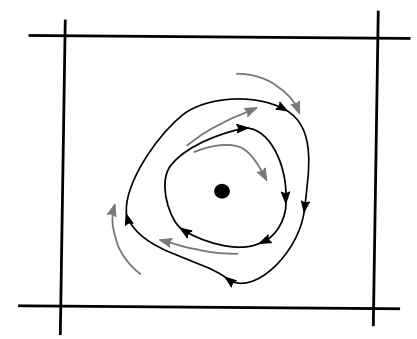

Figure 2: An $\frac{1}{n} \times \frac{1}{n}$-square which contains several attractors.

\subsection{Return $K_{e q}^{n}$.}

Properties $1 \& 3$ of the theorem are immediate. However, to have $K_{e q}^{n+1} \subseteq$ $K_{e q}^{n}$, there is a problem since for a $\frac{1}{n} \times \frac{1}{n}$ square $s$, it may happen that a $\frac{1}{n+1} \times \frac{1}{n+1}$ square overlaps partially $s$ and its complement, and this square includes a zero of $f$. To prevent this situation, it is enough to substitute $n_{0}=2^{n}$ in line 1 of the algorithm above.

We have the following corollary.

Corollary 22 Given as input $\rho$-names of $f$ and of a compact subset $K \subseteq \mathbb{R}^{2}$, one can semidecide whether or not it contains at least one equilibrium point of (1). In other words, there is an algorithm that, given as input $\rho$-names of $f$ and $K$, tells in a finite amount of time if $f$ does not have a zero in $K$, but needs an infinite amount of time to conclude that $K$ includes a zero of $f$.

\subsection{Computing hyperbolic periodic orbits}

In this section, we show how to compute attracting hyperbolic periodic orbits in structurally stable systems. By a time transformation $\bar{t}=-t$ the same algorithm can be used to compute repelling hyperbolic periodic orbits. The idea behind this algorithm is to use the construction of Section 4 and the fact that, in a sufficiently small neighborhood, the flow will converge to a given attracting hyperbolic periodic orbit exponentially, i.e. convergence is bounded by a term $\beta e^{-\lambda t}$ with $\beta>0$ and $\lambda>0$.

Our construction is made modulo $\frac{1}{n} \times \frac{1}{n}$-squares. This means that within $\frac{1}{n} \times \frac{1}{n}$-squares one is not able to distinguish objects. For instance, as Fig. 2 depicts, it may happen that a $\frac{1}{n} \times \frac{1}{n}$-square which seems containing one sink contains in fact two hyperbolic periodic orbits or that an $n$-polygonal periodic orbit, as defined below, contains several hyperbolic periodic orbits.

Definition 23 Assume the conditions of Section 4. An n-polygonal periodic orbit is a sequence of $\frac{1}{n} \times \frac{1}{n}$-squares $s_{1}, \ldots, s_{k}$ such that:

1. $s_{i+1} \neq s_{i}$ for $i=1 \ldots, k-1$. 


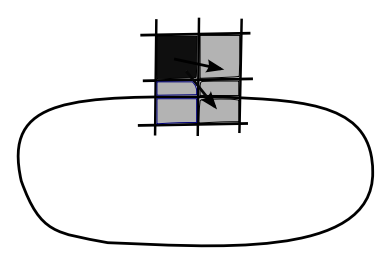

Figure 3: Computing a hyperbolic attracting periodic orbit.

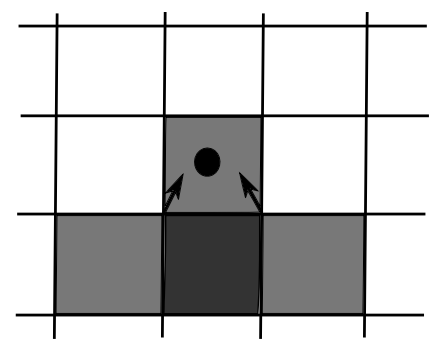

Figure 4: The selected $\frac{1}{n} \times \frac{1}{n}$-square $s$ below the sink is seen in more detail in Fig. 5. The polygon of direction is computed over the whole square, so the simulated dynamics push $\operatorname{Traj}_{n}(s)$ wrongly to the side $\frac{1}{n} \times \frac{1}{n}$-squares, allowing the existence of $n$-polygonal periodic orbits.

2. $s_{i+1} \in \Theta\left(s_{i}\right), i=1,2, \ldots, k-1$, and $s_{1} \in \Theta\left(s_{k}\right)$.

Formally, condition 1 is not needed as $s_{i} \notin \Theta\left(s_{i}\right)$; it is included for clarity. Notice that any $n$-polygonal periodic orbit is a connected set and divides $S_{n}$ into two regions, one "outside" the $n$-polygonal periodic orbit, and one inside (one of these sets may be empty).

As expected, $n$-polygonal periodic orbits will be used to cover hyperbolic attracting periodic orbits. In particular, if $S_{n}$ contains a periodic orbit $\gamma$ as well as a sufficiently small neighborhood $N_{\varepsilon}(\gamma)$ of $\gamma$ where the flow converges to the orbit exponentially, then for any $\frac{1}{n} \times \frac{1}{n}$-square $s \subseteq N_{\varepsilon}(\gamma)$ with sufficiently large $n, \operatorname{Traj}_{n}(s) \subseteq N_{\varepsilon}(\gamma)$ and it is not marked by $\boldsymbol{\alpha}$ (the error committed when computing $\Theta(s)$, or more precisely, when computing $f(s)$, bounded by $\frac{1}{n}$, is sufficiently small to be accommodated within the exponential factor) - see Fig. 3.

A potential problem in this approach is that we may get "fake" periodic orbits near some sinks. It may happen that, when near a sink, we get some $n$ polygonal periodic orbits and we are unable to tell whether or not they contain periodic orbits, as Fig. 4 and Fig. 5 suggest. For this reason, we need to refine the construction of Section 4.

The problem is rooted at the construction that $f(s)$, thus $P_{s, n}$, is computed all over $s$, which may lead to an excessive overestimation of $\Theta(s)$ (see Fig. 

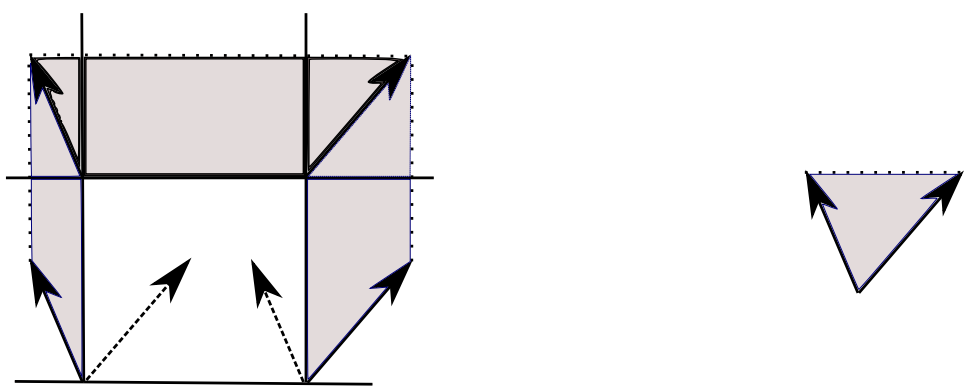

Figure 5: The selected $\frac{1}{n} \times \frac{1}{n}$-square $s$ below the sink of Fig. 4 (left side). On the right side one can find the polygon of directions computed over $s$.
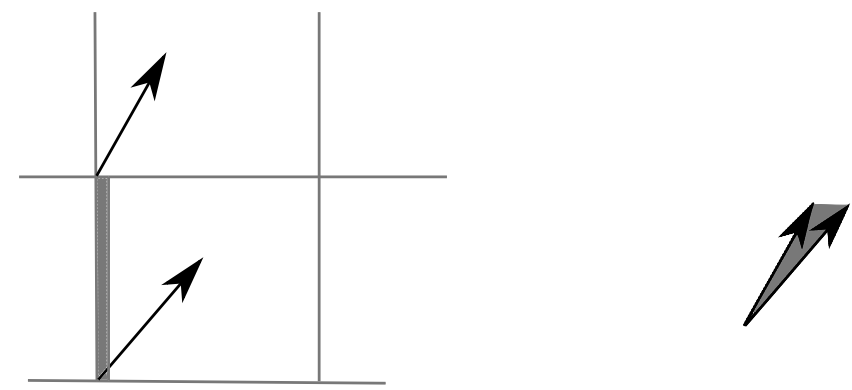

Figure 6: A refined version of $\Theta(s)$, now computed for each face. Near this face we build an $\frac{1}{n} \times \frac{1}{n^{2}}$-square and compute a polygon of directions over it (right side) to refine $\Theta(s)$.

5). To avoid this problem, we refine the construction in the following manner: adjacent to each face $\alpha$ of $s$ (and inside $s$ ), we build an $\frac{1}{n} \times \frac{1}{n^{2}}$-rectangle $r_{\alpha}$ as Fig. 6 depicts and similarly to the construction of Section 4, we compute a rational polytope $P_{s, n}^{\alpha}$ (polytope of directions) such that $f\left(r_{\alpha}\right) \subseteq P_{s, n}^{\alpha}$ and $\operatorname{dist}\left(f\left(r_{\alpha}\right), P_{s, n}^{\alpha}\right)<\frac{1}{n^{2}}$. We then define

$$
\tilde{\Theta}(s)=\bigcup_{\alpha \text { is a face of } s} A_{\alpha},
$$

where $A_{\alpha}$ is the collection of all $\frac{1}{n} \times \frac{1}{n}$ squares which are adjacent to the face $\alpha$ of $s$ (not including $s$ ) and intersect with the following rational polytope

$$
R_{s, n}^{\alpha}=\left\{\alpha+t P_{s, n}^{\alpha} \mid t \in \mathbb{R}_{0}^{+}, \alpha \text { is a face of } s\right\} .
$$

It is easy to see that $\tilde{\Theta}(s) \subseteq \Theta(s)$, i.e. $\tilde{\Theta}(s)$ is a refinement of $\Theta(s)$, and that Lemmas 18 and 19 still hold. More importantly, $\tilde{\Theta}(s)$ avoids fake periodic orbits immediately surrounding sinks. Indeed, near a face $\alpha, R_{s, n}^{\alpha}$ will give the flow 
with an error bounded by $\frac{1}{n^{2}}$, which as $n \rightarrow+\infty$ converges towards the dynamics of the flow in this face, irrelevant to what happens elsewhere in $s$. Hence fake flows no longer exist in a sufficiently small neighborhood of any sink. This is important for determining the domain of attraction of a sink, because if possible limit cycles exist in its surroundings (as "fake" $n$-polygonal periodic orbits like those in Fig. 4 may suggest), we have to consider the possibility that the flow is converging towards a periodic orbit other than towards the sink, and hence the previous algorithm cannot reliably determine the domain of attraction of a sink. Therefore, when computing the dynamics of the flow, we will use $\tilde{\Theta}$ instead of $\Theta$ (e.g. when computing $\operatorname{Traj}_{n}(s)$ ).

If one has an $n$-polygonal periodic orbit $\Gamma$ such that $\operatorname{Traj}_{n}(\Gamma)$ is not marked with $\boldsymbol{q}$ and does not include an equilibrium point (this can be semidecided by Corollary 22), then $\Gamma$ must contain at least one hyperbolic attracting periodic orbit. On the other hand, by the facts mentioned above, for any hyperbolic attracting periodic orbit and for $n$ big enough, there is an $n$-polygonal periodic orbit $\Gamma$ such that $\operatorname{Traj}_{n}(\Gamma)$ is not marked with and for this $n$, the algorithm of Section 5.1 excludes the possibility of having an equilibrium point in $\Gamma$ using Corollary 22.

Thus, to compute hyperbolic attracting periodic orbits, we should perform, for each $n$, the following algorithm:

1. For every $\frac{1}{n} \times \frac{1}{n}$-square $s$ in $S_{n}$, compute $\operatorname{Traj}_{n}(s)$.

2. Check if $\operatorname{Traj}_{n}(s)$ contains any $n$-polygonal periodic orbit. If yes, for every such $n$-polygonal periodic orbit $\Gamma$ check if $\operatorname{Traj}_{n}(\Gamma)$ is marked with $\boldsymbol{\alpha}$ or if it includes an equilibrium. If the answer is:

(a) Yes, then $\Gamma$ may or may not include a hyperbolic attracting periodic orbit.

(b) No, then $\Gamma$ includes at least one hyperbolic attracting periodic orbit.

For every $n$, each hyperbolic attracting periodic orbit inside $S_{n}$ is always covered by an $n$-polygonal periodic orbit $\Gamma$. Notice also that, within a fixed compact set, if we denote the union of all $n$-polygonal periodic orbits as $P_{n}$, then the area of $P_{n}$ converges to 0 as $n \rightarrow \infty$. On the other hand, for any $n$ polygonal periodic orbit $\Gamma$ with $n$ sufficiently large, we can determine whether or not $\Gamma$ actually contains an hyperbolic attracting periodic orbit since, within every fixed compact set, as $n \rightarrow+\infty$, all $n$-polygonal periodic orbits not covering a hyperbolic attracting periodic orbit will become broken. Therefore we can semidecide the presence of hyperbolic attracting periodic orbits in a compact subset of $\mathbb{R}^{2}$. The following result makes this more precise.

Theorem 24 Assume that $f \in C^{1}\left(\mathbb{R}^{2}\right)$ and that the system (1) is structurally stable. Let $K \subseteq \mathbb{R}^{2}$ be a computable compact set and let $K_{\text {cycles }}$ be the union of all hyperbolic periodic orbits of (1) contained in $K$. Then, given as input $\rho$-names of $f$ and $K$, one can compute a sequence of closed sets $\left\{K_{c y c l e s}^{n}\right\}_{n \in \mathbb{N}}$ with the following properties: 
1. $K_{\text {cycles }}^{n} \subseteq K$ for every $n \in \mathbb{N}$.

2. $K_{\text {cycles }}^{n+1} \subseteq K_{\text {cycles }}^{n}$ for every $n \in \mathbb{N}$.

3. $\lim _{n \rightarrow \infty} K_{\text {cycles }}^{n}=K_{\text {cycles }}$.

Proof. The proof follows from the argument above, using a similar method as that in the proof of Theorem 21.

Corollary 25 Given as input $\rho$-names of $f$ and of a compact $K \subseteq \mathbb{R}^{2}$, one can semidecide if it contains at least one hyperbolic periodic orbit of (1). In other words, there is an algorithm that given as input $\rho$-names of $K$ and $f$ will tell in a finite amount of time if $f$ has a hyperbolic periodic orbit of (1) in $K$, but may need an infinite amount of time to conclude that $K$ does not contain such an orbit.

Another related result is as follows.

Theorem 26 Given as input a $\rho$-name of an analytic function $f$, the problem of deciding the number of periodic orbits in (1) is undecidable, even on compact sets.

Proof. Consider the function $g$ defined in the proof of Theorem 20, and consider the computable system given in polar coordinates

$$
\left\{\begin{array}{l}
r^{\prime}=\left(r-\sum_{i=1}^{\infty} \frac{g(k, i)}{2^{2}}\right)(r-1) \\
\theta^{\prime}=1
\end{array}\right.
$$

for a fixed $k \in \mathbb{N}_{0}$, which can easily be converted to an equivalent system in rectangular coordinates. If $T M_{k}$ does not halt on input $k, \sum_{i=1}^{\infty} \frac{g(k, i)}{2^{i}}=1$, and the system (5) has only one periodic orbit $\gamma$, attracting inside $\gamma$ and repelling outside $\gamma$, which is a non-hyperbolic limit cycle. If $T M_{k}$ halts on input $k$, then $\sum_{i=1}^{\infty} \frac{g(k, i)}{2^{i}}<1$, and subsequently the system (5) has two hyperbolic periodic orbits, both centered at the origin: one attracting with radius $\sum_{i=1}^{\infty} \frac{g(k, i)}{2^{i}}$, and the other repelling with radius 1 . Thus we have reduced the problem of knowing the number of periodic orbits to the undecidable problem "does $T M_{k}$ halts on input $k$ ?", implying that the former problem is also undecidable.

Again, in this proof, we make critical use of the fact that the system can be unstable, thus allowing that two hyperbolic periodic orbits collapse to a nonhyperbolic one. Other types of bifurcations could be used to recreate undecidability of the previous problem, e.g. Hopf bifurcations, in which a sink becomes a non-hyperbolic asymptotically stable equilibrium at bifurcation, which transforms itself to a source plus a limit cycle after bifurcation. This happens, for instance, to the system

$$
\left\{\begin{array}{l}
x^{\prime}=y \\
y^{\prime}=\left(\alpha-x^{2}\right) y-x
\end{array}\right.
$$


described in [HW95, Example 9.2.2], where a Hopf bifurcation happens for $\alpha=$ 0 .

It would be interesting to know if the previous problem becomes decidable for structurally stable systems.

We mention in passing that the previous result is related to Hilbert's 16th problem: determine an upper bound for the number of limit cycles in a planar system

$$
\begin{aligned}
& x^{\prime}=P(x, y) \\
& y^{\prime}=Q(x, y)
\end{aligned}
$$

where $P$ and $Q$ are polynomials. The previous result shows that this cannot be done algorithmically, though we believe that for really solving the problem, one should add some stability conditions to (6) so that the problem is meaningful, namely structural stability.

\subsection{Computing domains of attraction}

In this section, we investigate the question of computing the domains of attraction of a given attractor. However, in practical applications, it is usually more important to know that a certain compact set $C$ indeed contains one or more attractors, and to determine the union of domains of all attractors in $C$. This is of interest, for example, to control theory. For instance, imagine that we are dealing with a nuclear reactor and want to decide the set of initial conditions leading up to a critical domain of operation (i.e. these are points to be avoided). We pick a compact set that covers all these critical situations and then determine the domain of attraction as described above within this compact set. This is the approach that we will use in this paper.

Usually it is assumed that $C$ is invariant, but this assumption is not necessary. The set $C$ may have some parts to which the flow circulates before reaching attractors outside of $C$. This does not pose a problem and henceforth we do not assume that $C$ is invariant.

As a first case, let $\mathcal{S}\left(\mathbb{R}^{2}\right)$ be the set of $C^{1}$ structurally stable vector fields on $\mathbb{R}^{2}, f \in \mathcal{S}\left(\mathbb{R}^{2}\right)$, and $A_{f}$ the class of compact sets $A \subseteq \mathbb{R}^{2}$ containing at least one hyperbolic attractor (in this case, sink or attracting periodic orbit) of (1) such that no limit set of (1) intersects simultaneously $A$ and its complement. Note that $\mathcal{S}\left(\mathbb{R}^{2}\right) \subseteq C^{1}\left(\mathbb{R}^{2}\right)$ and that functions in $C^{1}\left(\mathbb{R}^{2}\right)$ admit names, e.g. by means of rational polygonal functions over increasing rational cubes over $\mathbb{R}^{2}$ see [Wei00] for details.

The following result shows that, given dynamics of (1), the operator that gives the basin of attractors in $A$ is semi-computable.

Theorem 27 Assume the conditions as above. The function $F:\{(A, f) \mid f \in$ $\left.\mathcal{S}\left(\mathbb{R}^{2}\right), A \in A_{f}\right\} \rightarrow \mathcal{O}\left(\mathbb{R}^{2}\right)$, where $F(A, f)=$ union of domains of all attractors of (1) in $A$, is semi-computable, but not computable. 
Proof. (Sketch) The fact that $F$ is not computable follows from a result of Zhong [Zho09], which shows that there is a computable $C^{\infty}$ function $f$ such that (1) has a sink at the origin whose domain of attraction is not computable (if $F$ is computable then this domain of attraction would be computable).

To prove the semi-computability part, we use the more accurate function $\tilde{\Theta}$ of Section 5.2 instead of the function $\Theta$ of Section 4. We first observe that for any point $x$ in the interior of $F(A, f)$ whose trajectory does not converge to the boundary of $F(A, f)$ (i.e. part of the boundary is not itself an attractor - we will see that this condition is of uttermost importance for the theorem to hold), the $n$-polygonal trajectory of any square $s$ containing $x$ will converge to a sink or a hyperbolic attracting periodic orbit contained in $A$ for $n$ large enough (making use of Lemma 10, the fact that attractors inside $A$ are stable, and the hypothesis that $\Gamma_{x}^{+}$does not converge to the boundary of the domain of attraction. The only other problem that may occur is that $s$ crosses a hyperbolic repelling periodic orbit, but this can be checked by performing the time transformation $\bar{t}=-t$, as mentioned in Section 5.2, together with the fact that the area of squares intersecting these repelling orbits tends to 0 in a given compact set as $n \rightarrow+\infty$ ). Using the results of Section 5, we can locate the regions where equilibria and hyperbolic periodic orbits lie. Therefore, for any $\frac{1}{n} \times \frac{1}{n}$-square $s$ with $\operatorname{Traj}_{n}(s)$ not marked with $\boldsymbol{p}$, there are three scenarios: (a) if $\operatorname{Traj}_{n}(s)$ does not reach $A$, then $s$ definitely does not belong to $F(A, f)$, (b) if $\operatorname{Traj}_{n}(s)$ reaches $A$ but does not intersect any other located regions containing attractors other than those in $A$ (thus $\operatorname{Traj}_{n}(s)$ does not include any attractor besides those in $A$ ), then $s \subset F(A, f)$, and (c) if $\operatorname{Traj}_{n}(s)$ goes to $A$, but also intersect located regions containing other equilibria/hyperbolic periodic orbits. In this case, we have to wait for an $n$ big enough to distinguish the convergence towards $A$ or to attractors outside of $A$. If $\operatorname{Traj}_{n}(s)$ is marked with $\mathbf{p}$, then we cannot tell whether or not $s$ belongs to the domain of attractors in $A$.

Therefore, for a given $n$, we mark a $\frac{1}{n} \times \frac{1}{n}$-square $s$ in $S_{n}$ to indicate $s \subset$ $F(A, f)$ only if $\operatorname{Traj}_{n}(s)$ is not marked with $\boldsymbol{\phi}, \operatorname{Traj}_{n}(s) \cap A \neq \varnothing$, and $\operatorname{Traj}_{n}(s)$ does not include any attractor besides those in $A$. As $n \rightarrow+\infty$, the set of marked $\frac{1}{n} \times \frac{1}{n}$-squares will fully cover the domain of attraction of $A$.

The previous result no longer is true if we allow all $C^{1}$ functions $f$, instead of only of those who give structurally stable systems.

Theorem 28 Assume the conditions as above, the function $F:\left\{\left(A_{f}, f\right) \mid f \in\right.$ $\left.C^{1}\left(\mathbb{R}^{2}\right)\right\} \rightarrow \mathcal{O}\left(\mathbb{R}^{2}\right)$ defined by $F(A, f)=$ domain of attraction of $A$ for (1) is not semi-computable, even if restricted to use analytic functions.

Proof. Consider the following system, defined by polar coordinates

$$
\left\{\begin{array}{l}
r^{\prime}=-r\left[\left(r^{2}-1\right)^{2}-\mu\right] \\
\theta^{\prime}=1
\end{array}\right.
$$

adapted from [Per01, p. 319, Example 2], which can easily be written in $(x, y)$ coordinates. When $\mu<0$, the system has just one attractor, the fixed point 0 (see Fig. 7). When $\mu=0$, the point 0 still is a sink, but a cycle, with center 

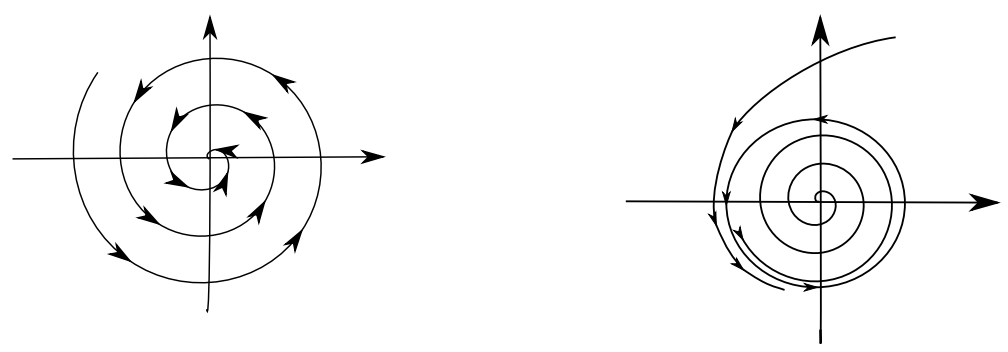

Figure 7: Phase portrait for $\mu<0$ (left) and $\mu=0$ (right)

at 0 and radius 1 appears (see Fig. 7). Therefore the domain of attraction of the sink at 0 is $\mathbb{R}^{2}$ for $\mu<0$, and $\left\{(x, y) \in \mathbb{R}^{2} \mid x^{2}+y^{2}<1-\sqrt{\mu}\right\}$ for $\mu \geq 0$. Therefore the map $F$ is not continuous over its domain, and since computable functions are continuous [Wei00], $F$ cannot be computable (semi-computability is in essence computability with respect to $\rho$-names for open sets formed by a subbasis of the topology - so semi-computable functions are still continuous).

It is not hard to see that our previous construction does not work when $\mu=0$ in the example of Fig. 7. Each time a square outside the cycle approaches the latter, it will eventually overlap the cycle and our square will overlap the domain of attraction of 0 and will falsely be drawn to this equilibrium. Therefore we cannot compute the domain of attraction of the equilibrium point 0 .

As a corollary of Theorem 27, we obtain a result already stated in [Zho09], but with a different proof and for structurally stable systems.

Corollary 29 Assume the system (1) is structurally stable and that $x_{0}$ is a sink. If $f$ is computable and analytic, then the domain of attraction of $x_{0}$ is an r.e. open set of $\mathbb{R}^{2}$.

Acknowledgments. D. Graça was partially supported by Fundação para a Ciência e a Tecnologia and EU FEDER POCTI/POCI via CLC, SQIG - Instituto de Telecomunicações. DG was also attributed a Taft Research Collaboration grant which made possible a research visit to U. Cincinnati. N. Zhong was partially supported by the 2009 Taft Summer Research Fellowship.

\section{References}

[AC84] Jean-Pierre Aubin and Arrigo Cellina. Differential inclusions: Setvalued maps and viability theory. Number 364 in Grundlehren der Mathematischen Wissenschaften. Springer-Verlag, Berlin, 1984.

[BBKT01] V. D. Blondel, O. Bournez, P. Koiran, and J. N. Tsitsiklis. The stability of saturated linear dynamical systems is undecidable. $J$. Comput. System Sci., 62:442-462, 2001. 
[BGZed] J. Buescu, D. S. Graça, and N. Zhong. Computability and dynamical systems. In Dynamics, Games and Sicence. Submitted.

[BY06] M. Braverman and M. Yampolsky. Non-computable Julia sets. J. Amer. Math. Soc., 19(3):551-0578, 2006.

[CG09] P. Collins and D. S. Graça. Effective computability of solutions of differential inclusions - the ten thousand monkeys approach. Journal of Universal Computer Science, 15(6):1162-1185, 2009.

[Col05] P. Collins. Continuity and computability of reachable sets. Theor. Comput. Sci., 341:162-195, 2005.

[Dei84] Klaus Deimling. Multivalued differential equations. Number 1 in de Gruyter Series in Nonlinear Analysis and Applications. Walter de Gruyter \& Co, Berlin, 1984.

[GCB09] D. S. Graça, M. L. Campagnolo, and J. Buescu. Computational bounds on polynomial differential equations. Appl. Math. Comput., 215(4):1375-1385, 2009.

[GH83] J. Guckenheimer and P. Holmes. Nonlinear Oscillations, Dynamical Systems, and Bifurcation of Vector Fields. Springer, 1983.

[GZ09] D. S. Graça and N. Zhong. Computing domains of attraction for planar dynamics. In C. S. Calude, J. F. Costa, N. Dershowitz, E. Freire, and G. Rozenberg, editors, 8th International Conference on Unconventional Computation (UC 2009), LNCS 5715, pages 179190. Springer, 2009.

[GZB09] D.S. Graça, N. Zhong, and J. Buescu. Computability, noncomputability and undecidability of maximal intervals of IVPs. Trans. Amer. Math. Soc., 361(6):2913-2927, 2009.

[Hoy07] M. Hoyrup. Dynamical systems: stability and simulability. Math. Structures Comput. Sci., 17:247-259, 2007.

[HS74] M. W. Hirsch and S. Smale. Differential Equations, Dynamical Systems, and Linear Algebra. Academic Press, 1974.

[HW95] J. H. Hubbard and B. H. West. Differential Equations: A Dynamical Systems Approach - Higher-Dimensional Systems. Springer, 1995.

[Ko91] K.-I Ko. Computational Complexity of Real Functions. Birkhäuser, 1991.

[Lor63] E. N. Lorenz. Deterministic non-periodic flow. J. Atmos. Sci., 20:130-141, 1963.

[Moo90] C. Moore. Unpredictability and undecidability in dynamical systems. Phys. Rev. Lett., 64(20):2354-2357, 1990. 
[Odi89] P. Odifreddi. Classical Recursion Theory, volume 1. Elsevier, 1989.

[PBV95] A. Puri, V. Borkar, and P. Varaiya. Epsilon-approximation of differential inclusions. In Proc. of the 34th IEEE Conference on Decision and Control, pages 2892-2897, 1995.

[PER89] M. B. Pour-El and J. I. Richards. Computability in Analysis and Physics. Springer, 1989.

[Per01] L. Perko. Differential Equations and Dynamical Systems. Springer, 3rd edition, 2001.

[RWZ09] R. Rettinger, K. Weihrauch, and N. Zhong. Topological complexity of blowup problems. Journal of Universal Computer Science, 15(6):1301-1316, 2009.

[Via00] M. Viana. What's new on Lorenz strange attractors? Math. Intelligencer, 22(3):6-19, 2000.

[Wei00] K. Weihrauch. Computable Analysis: an Introduction. Springer, 2000 .

[Zho09] N. Zhong. Computational unsolvability of domain of attractions of nonlinear systems. Proc. Amer. Math. Soc., 137:2773-2783, 2009.

[ZW03] N. Zhong and K. Weihrauch. Computability theory of generalized functions. Journal of the ACM (JACM), 50(4):469-505, 2003. 